ARTICLE

DOI: $10.1038 / s 41467-018-06407-7$

\title{
An OB-fold complex controls the repair pathways for DNA double-strand breaks
}

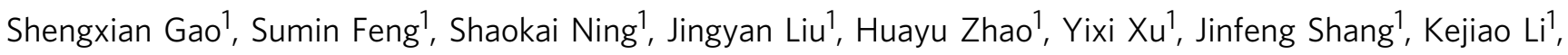
Qing Li (i) ${ }^{1}$, Rong Guo ${ }^{1} \&$ Dongyi Xu (i) ${ }^{1}$

53BP1 with its downstream proteins, RIF1, PTIP and REV7, antagonizes BRCA1-dependent homologous recombination (HR) and promotes non-homologous end joining (NHEJ) in an unclear manner. Here we show that REV7 forms a complex with two proteins, FAM35A and C20ORF196. We demonstrate that FAM35A preferentially binds single-strand DNA (ssDNA) in vitro, and is recruited to DSBs as a complex with C20ORF196 and REV7 downstream of RIF1 in vivo. Epistasis analysis shows that both proteins act in the same pathway as RIF1 in NHEJ. The defects in HR pathway to repair DSBs and the reduction in resection of broken DNA ends in BRCA1-mutant cells can be largely suppressed by inactivating FAM35A or C20ORF196, indicating that FAM35A and C20ORF196 prevent end resection in these cells. Together, our data identified a REV7-FAM35A-C200RF196 complex that binds and protects broken DNA ends to promote the NHEJ pathway for DSB repair.

\footnotetext{
${ }^{1}$ State Key Laboratory of Protein and Plant Gene Research, School of Life Sciences, Peking University, 100871 Beijing, China. These authors contributed equally: Shengxian Gao, Sumin Feng. Correspondence and requests for materials should be addressed to R.G. (email: guorong@pku.edu.cn) or to D.X. (email: xudongyi@pku.edu.cn)
} 
M ammalian cells utilize two major pathways to repair double-strand breaks (DSBs), which are the main source of DNA damage and must be repaired to allow cells to survive. One pathway depends on homologous recombination (HR), whereas the other one uses non-homologous end joining (NHEJ) $)^{1,2}$. Resection of DNA ends, which converts broken DNA into $5^{\prime}$-recessed ends suitable for HR, is a key reaction by which the selection of the pathway is controlled ${ }^{1,2}$. BRCA 1 promotes this reaction and thus stimulates the HR pathway ${ }^{3}$, whereas 53BP1 and its downstream proteins, $\mathrm{RIF}^{4-8}, \mathrm{PTIP}^{9}$ and REV7 ${ }^{10,11}$, suppress the reaction and thus facilitate the NHEJ pathway. However, how the 53BP1-RIF1-REV7 axis suppresses the resection is unclear.

Here, we identify a REV7 complex with FAM35A and C20ORF196, which is distinct from the Polל complex of REV7. FAM35A resembles a telomere-protecting protein, POT1, in that both proteins contain three OB-fold domains and bind singlestrand DNA (ssDNA). Cells lacking either FAM35A or C20ORF196 are largely defective in NHEJ pathway, but not HR pathway. Interestingly, the absence of FAM35A or C20ORF196 rescues HR and end resection in the BRCA1-deficient cells. Thus, our data identify a REV7-FAM35A-C20ORF196 complex that protects broken DNA ends to antagonize BRCA1-dependent resection, and its mode of action may resemble that of POT1.

\section{Results}

FAM35A and C20ORF196 form a complex with REV7. To investigate the mechanism of REV7 in protecting DSB ends, we immunopurified its complexes from HEK293 cells transiently expressing FLAG-REV7 with anti-FLAG antibody. Mass spectrometry analysis revealed that two proteins, FAM 35A and C20ORF196, co-immunoprecipitated with REV7 (Fig. 1a, Table 1 and Supplementary Data 1). Immunoblotting confirmed this finding (Fig. 1b). Immunoblotting and mass spectrometry analysis of reciprocal immunoprecipitation using HEK293 cells expressing FLAG-tagged FAM35A and C20ORF196 showed that REV7, FAM35A and C20ORF196 are mutually present in their immunoprecipitates (Fig. 1a-d, Table 1 and Supplementary Data 1). REV7 is one component of the Pol complex $^{12}$. Mass spectrometry analysis of the immunoprecipitated mixtures and immunoblotting revealed that the Pol $\zeta$ subunits, REV3L, POLD2 and POLD3 existed in the complexes of REV7 but not those of FAM35A and C20ORF196 (Fig. 1a-d, Table 1 and Supplementary Data 1), suggesting that REV7 forms two distinct complexes:

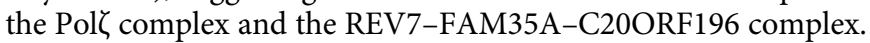

To examine the direct interactions in the REV7-FAM35A-C20ORF196 complex, we performed MBPand GST-pulldown assays with recombinant proteins. Results showed that MBP-FAM35A pulled-down both C20ORF196 and REV7 (Fig. 1e, f), and GST-REV7 also brought-down C20ORF196 (Fig. 1g), suggesting that these three proteins are able to interact directly with each other.

In addition to REV7, FAM35A and C20ORF196, mass spectrometry also identified RIF1 in all the immunoprecipitates of these three proteins (Table 1 and Supplementary Data 1). Because RIF1 is a common contamination in immunoprecipitations ${ }^{13}$, we examined this finding carefully under a condition with ethidium bromide $(\mathrm{EtBr})$, which impairs protein-DNA interaction. Immunoblotting confirmed that RIF1 was co-purified by FLAG-tagged REV7, FAM35A or C20ORF196 even with the presence of EtBr (Fig. 1b-d). These results suggest that RIF1 interacts with the REV7-FAM35A-C20ORF196 complex not through a bridge of DNA.
FAM35A prefers to bind ssDNA. FAM35A and C20ORF196 are expressed in most vertebrates and a few of invertebrates. Threedimensional structure prediction with HHprep (https://toolkit. tuebingen.mpg.de/\#/tools/hhpred) revealed that while C20ORF196 has no recognizable domains, FAM35A contains three OB-folds at its C-terminus, which resemble the structure of POT1, a component of the telomere protection complex (Fig. 1h and Supplementary Fig. 1a, b). It has been shown that many OBfolds are ssDNA-binding domains and/or protein-interacting domains ${ }^{14}$. In fact, POT1 has been shown to bind ssDNA regions of telomeric DNA through its OB-folds and this binding is required for telomere stability ${ }^{15}$. The similarity between FAM35A and POT1 prompted us to examine whether FAM35A may bind ssDNA and protect broken DNA ends. We expressed and purified a recombinant protein containing all three OB-fold domains (381-904 aa) fused to the maltose-binding protein (MBPFAM35A_C; Supplementary Fig. 2a, b). Gel-shift assay showed that MBP-FAM35A_C bound a long ssDNA substrate $(60 \mathrm{nt})$ but not a short one (30 nt; Fig. 2a, b). It also bound double-strand DNA (dsDNA; 60 bp) but with a much lower affinity than ssDNA (60 nt; Fig. 2a, b), with a $K_{\mathrm{d}}$ of $\sim 750 \mathrm{nM}$, compared with $\sim 320$ $\mathrm{nM}$ for $60 \mathrm{nt}$ ssDNA. We further carried out competition experiments and confirmed that MBP-FAM35A_C prefers to bind $60 \mathrm{nt}$ ssDNA compared with $30 \mathrm{nt}$ ssDNA and $60 \mathrm{bp}$ dsDNA (Fig. 2c, d).

FAM35A interacts with REV7 through its $\mathrm{N}$-terminal region. FLAG immunoprecipitation and MBP-pulldown showed that a conserved N-terminal region RBD (REV7-binding domain; 1-54 aa) is necessary and sufficient for its interaction with REV7 (Supplementary Fig. 3a-e). Mutation in two conserved prolines to alanines (AA, P14A/P17A) of RBD region completely disrupted its interaction with REV7 (Supplementary Fig. 3a, f). In contrast, deletion of the N-terminal region of FAM35A (OB1-3) did not affect its interaction with C20ORF196 (Supplementary Fig. 3a, g). The further deletion of OB1 (OB2-3) strongly reduced its interaction with C20ORF196, suggesting that OB1 contributes to this association. Moreover, deletion of both $\mathrm{OB} 1$ and $\mathrm{OB} 2(\mathrm{OB} 3)$ or OB3 (OB1-2) completely disrupted its interaction with C20ORF196, indicating that the region (OB2-3) containing OB2 and $\mathrm{OB} 3$ are required for the interaction between FAM35A and C20ORF196 (Supplementary Fig. 3a, g, h).

FAM35A and C20ORF196 were recruited to DNA damage sites. We observed that green fluorescent protein (GFP)-tagged FAM35A was recruited to laser-induced DNA damage sites, implicating that FAM35A may act directly at the site of DNA damage (Supplementary Figs. 3a, 4a). Although the GFPFAM35A_RBD displayed strong signals at DNA damage sites, RBD domain-deleted mutant FAM35A_ $\triangle \mathrm{RBD}$ and FAM35A_OB1-3 only showed very weak signals (Supplementary Figs. 3a, 4a), suggesting that FAM35A may be recruited to DNA damage sites by two different mechanisms: the majority of FAM35A may be recruited by its interaction with REV7 through the RBD domain; whereas the minority of FAM $35 \mathrm{~A}$ may be through its OB-folds domain-mediated binding to the ssDNA at the broken DNA ends. These two mechanisms cooperatively recruit FAM35A to DSB sites because lacking any of them leaded to reduced signals (Supplementary Fig. 4b). Moreover, these OBfolds were able to be recruited to DNA damage sites individually (Supplementary Figs. 3a, 4c). Consistent with our speculation, depletion of REV7 strongly reduced the recruitment of GFP-FAM35A to DNA damage sites (Supplementary Fig. 4d, e). 
a

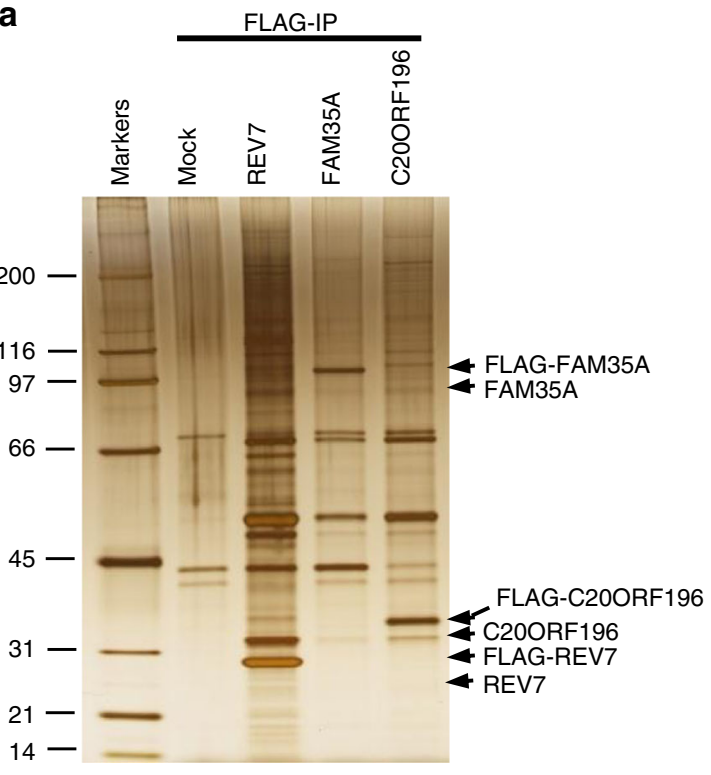

C

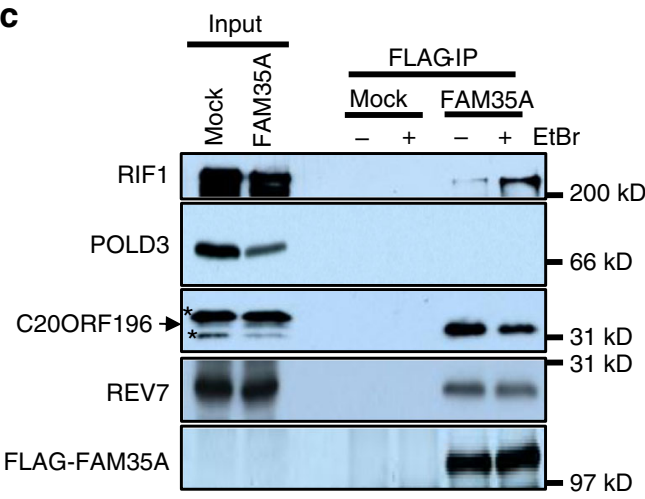

b

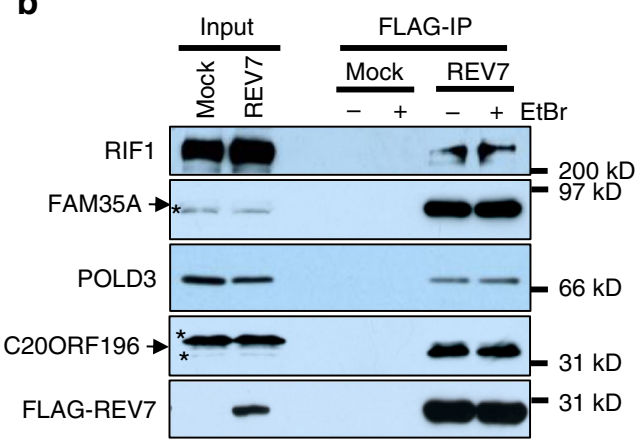

d

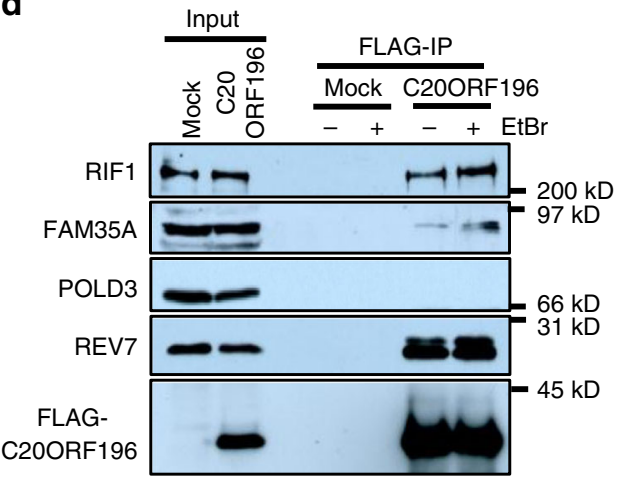

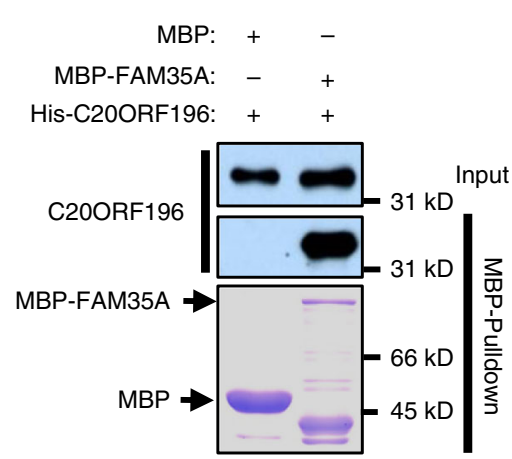

f

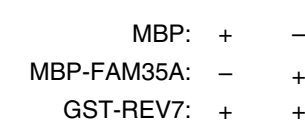

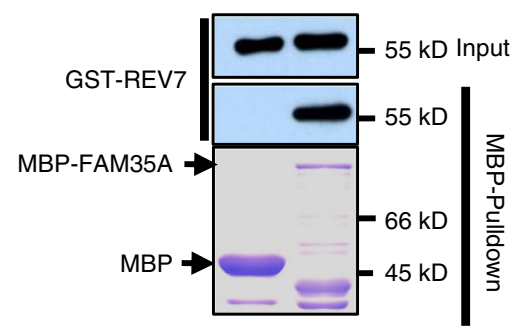

g

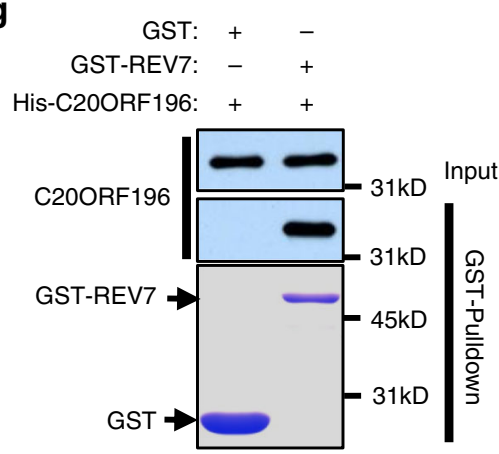

h

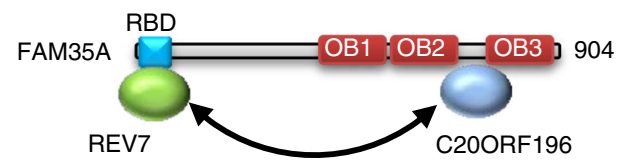

POT1 ᄃOB1: $\mathrm{OB} 2=\mathrm{OB} 3: \mathrm{HJR} \neg 634$

TPP1

Fig. 1 FAM35A and C20ORF196 form a complex with REV7. a Silver-stained SDS-PAGE gel showing the polypeptides that were immunopurified from extracts of HEK293 cells expressing FLAG-tagged REV7, FAM35A and C20ORF196 using the anti-FLAG antibody. The major polypeptides on the gel (arrows) were identified by mass spectrometry. b-d Immunoblot showing the IP of FLAG-tagged REV7 (b), FAM35A (c) and C200RF196 (d). IPs were performed with or without $100 \mu \mathrm{g} \mathrm{ml}^{-1} \mathrm{EtBr}$. The cross-reactive bands were indicated as asterisks. e-g MBP-pulldown (e, f) and GST-pulldown (g) examined the direct interactions among REV7, FAM35A and C20ORF196. The bait and prey proteins were detected with Coomassie blue staining and immunoblotting, respectively. $\mathbf{h}$ Schematic representation of FAM35A and POT1 
REV7 is recruited to DSB sites by RIF $1^{10,11}$. Consistently, the recruitment of the RBD region, but not the OB1-3, of FAM35A was strongly decreased when RIF1 is absent (Supplementary Fig. 4f-h). We further observed that GFP-C20ORF196 was also recruited to laser-induced DNA damage sites (Supplementary Fig. 5a), consistent with that it forms a complex with REV7 and FAM35A. Interestingly, the recruitment of REV7, FAM35A and C20ORF196 to DSB sites are interdependent (Supplementary Fig. 5a-g), implicating that they may be recruited as a complex. Moreover, RIF1 depletion also strongly reduced the recruitment

\begin{tabular}{|lllll|}
\hline \multirow{2}{*}{ Table 1 Peptide number identified by mass spectrometry } \\
Identified proteins & \multicolumn{1}{l}{ FLAG-IP } & & \\
\cline { 2 - 5 } & Mock & REV7 & FAM35A & C200RF196 \\
\hline REV7 & 0 & 25 & 5 & 3 \\
FAM35A & 0 & 3 & 88 & 12 \\
C20ORF196 & 0 & 0 & 5 & 20 \\
REV3L & 0 & 1 & 0 & 0 \\
POLD2 & 0 & 2 & 0 & 0 \\
POLD3 & 0 & 3 & 0 & 0 \\
RIF1 & 0 & 15 & 19 & 3 \\
\hline Full list of mass spectrometry data is available in Supplementary Data 1 \\
\hline
\end{tabular}
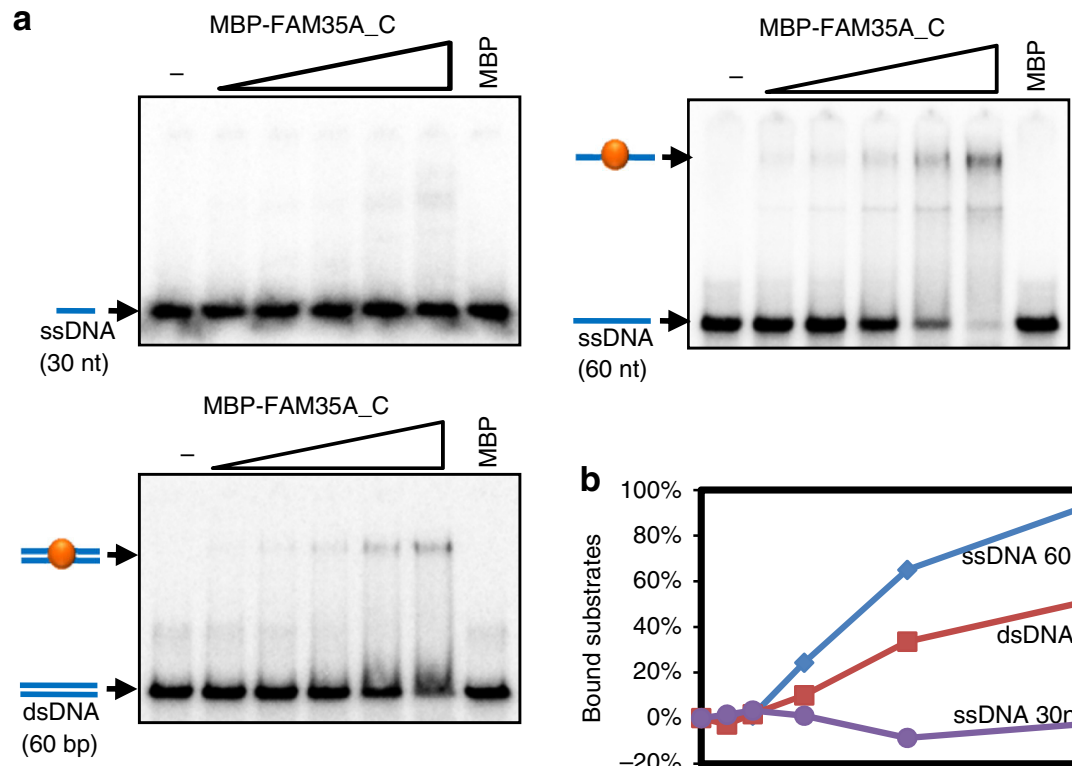

FAM35A and C20ORF196 promote NHEJ to repair DSB. To investigate the function of FAM35A and C20ORF196 in vivo, we generated the FAM35A or C20ORF196 null DT40 cells by gene targeting (Supplementary Fig. 6a-d). These cells displayed strong sensitivity to reagents that induce DSBs, including Bleomycin (a radiomimetic agent) and VP16 (etoposide, a Topoisomerase II [Topo2] inhibitor, which directly induces DSBs; Fig. 3a, b), demonstrating that FAM35A and C20ORF196 are required for normal DSB repair in vivo.

To examine which DSB repair pathway is defective in FAM35A -/- and C20ORF196 ${ }^{-/-}$cells, we utilized two drugs that induce distinct DSB damages that depend on a specific pathway for repair: ICRF193, which is a Topo2 inhibitor and induces DSB damages that specifically require the NHEJ pathway ${ }^{16-18}$; and $\mathrm{CPT}$, which is a TopoI inhibitor and induces one-end DSBs that depend on the HR pathway ${ }^{16-18}$. FAM $35 A^{-/-}$cells were hypersensitive to ICRF193, but not CPT (Fig. 3a), suggesting that FAM35A functions in NHEJ but not HR pathway. Other than hypersensitivity to ICRF193, C20ORF196-/- cells also showed mild, but reproducible, sensitivity to CPT (Fig. 3b), demonstrating that C20ORF196 has other function than NHEJ. NHEJ is essential for foreign DNA random integration in DT40

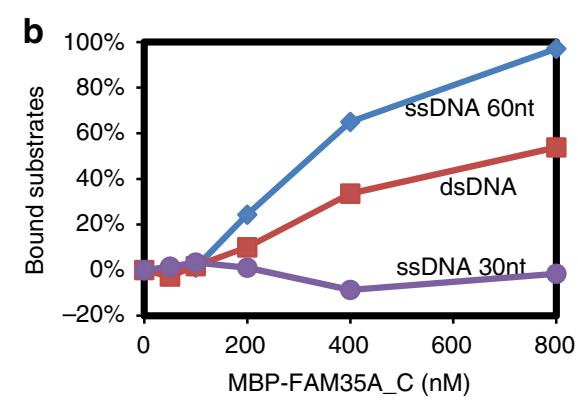

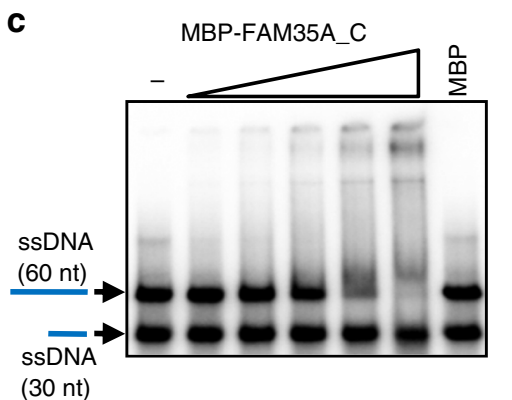

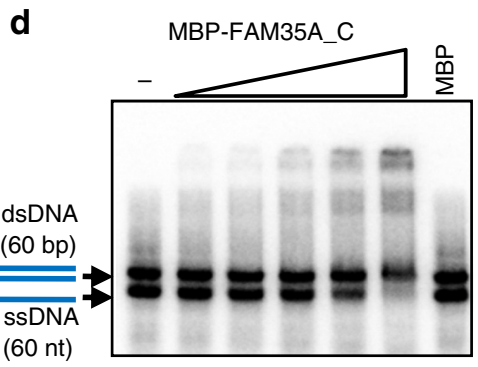

Fig. 2 FAM35A prefers to bind ssDNA. a, b Gel-shift assay (a) and its quantification (b) shows that MBP-FAM35A_C (381-904 aa) prefers to bind ssDNA. Reactions contained $5 \mathrm{nM}$ of the indicated ${ }^{32}$ P-labeled substrates and 0,50, 100, 200, 400 or $800 \mathrm{nM}$ purified MBP-FAM35A_C or $800 \mathrm{nM}$ MBP. c, d Competition experiments between 60 nt ssDNA and 30 nt ssDNA (c) or 60 bp dsDNA (d) for MBP-FAM35A_C binding 

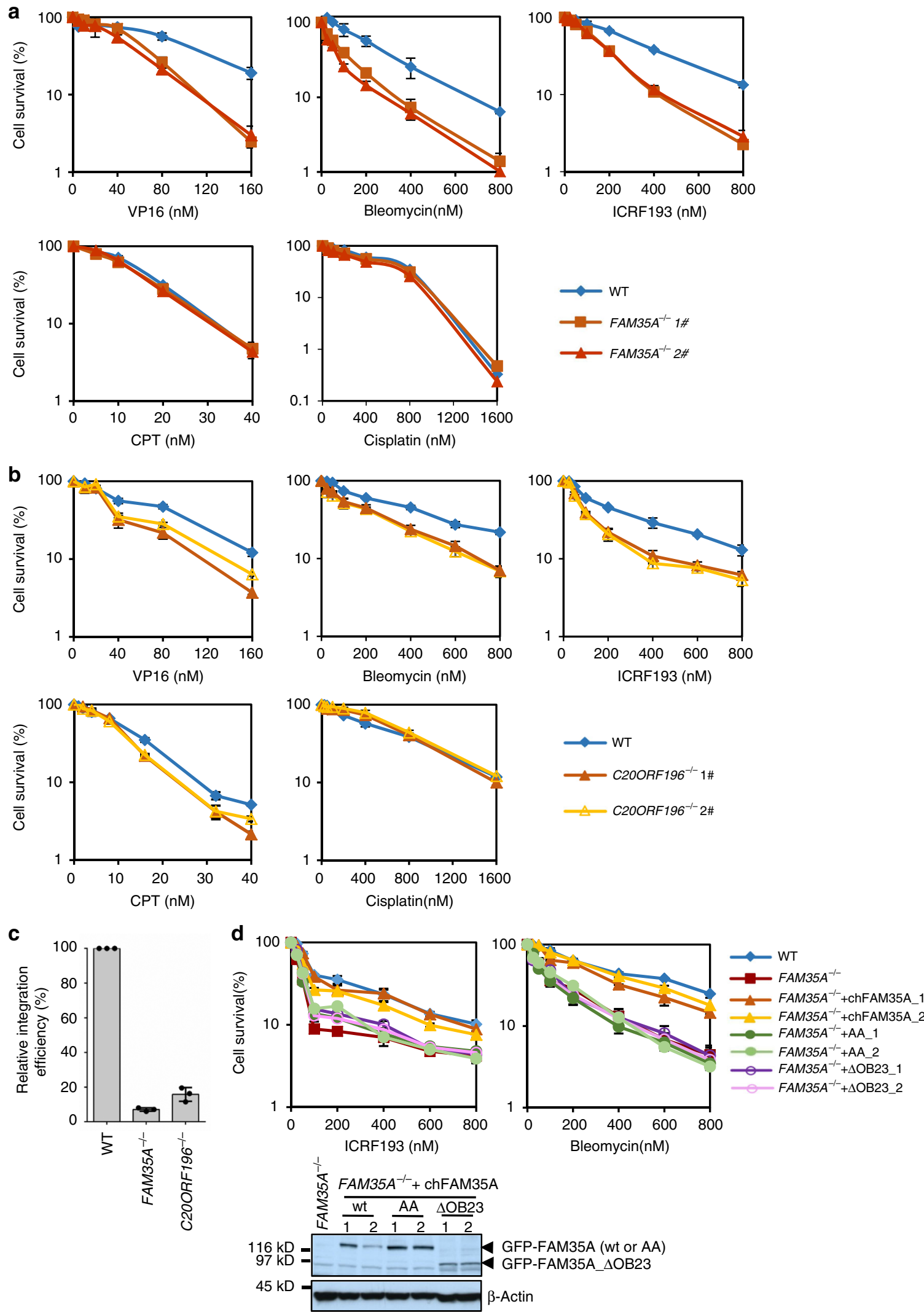

Fig. 3 FAM35A and C20ORF196 promote NHEJ to repair DSB. a, b Sensitivity assay of FAM35A (a) and C20ORF196 (b) knockout DT40 cells. The mean and s.d. from three independent experiments are shown. c Random integration assay of FAM35A and C200RF196 knockout DT40 cells. Cells were transfected with the pLoxPuro plasmid and clonogenic potential was determined in the presence of puromycin. Data are expressed as the mean number of puromycin resistant colonies \pm s.d. $(n=3)$. d Sensitivity assay of FAM35A-/- cells re-expressing GFP-fused wild-type or mutated chicken FAM35A. Protein expression levels of GFP-chFAM35A were shown by immunoblotting on the bottom 
cells $^{4,19}$. Consistently, random integration is decreased 14.2 and 6.3-folds in FAM $35 A^{-1-}$ and C20ORF196 ${ }^{-/-}$cells, respectively (Fig. 3c).

To examine which domain of FAM35A is required for its function in NHEJ, we carried out complementation experiments. Wild-type FAM35A, but not AA (P14A/P17A, which loses its interaction with REV7) and $\triangle \mathrm{OB} 23$ (1-576 aa, which loses interaction with C20ORF196 and ssDNA), restored the resistance to DSB-inducing drugs, ICRF193 and Bleomycin (Fig. 3d), demonstrating that both the interactions of FAM35A with REV7 and C20ORF196 or ssDNA are important for its function in NHEJ.

To check if FAM35A and C20ORF196 work in the same DSB repair pathway, we generated FAM $35 A^{-1-} C 20 O R F 196^{-/-}$double knockout cells and performed epistasis analysis. FAM $35 A^{-1-}$ C20ORF196-1- cells showed similar sensitivity to VP16, Bleomycin and ICRF193 as single knockout cells (Fig. 4a), demonstrating that FAM35A and C20ORF196 play a role in the same pathway to repair DSBs. REV7 suppresses end resection and promotes NHEJ downstream of RIF1 ${ }^{10,11}$. We examined if FAM35A and C20ORF196 also act in NHEJ in the same pathway with RIF1. FAM $35 A^{-/-}$RIF1 ${ }^{-/}$cells showed no more sensitivity to DSB-inducing reagents than single knockout cells (Fig. 4b), demonstrating that FAM35A acts in the same pathway as does RIF1. We thus concluded that REV7 forms a complex with FAM35A and C20ORF196 to repair DSBs through NHEJ.

REV7 is one subunit of $\mathrm{Pol} \zeta$, which is required for DNA interstrand crosslink (ICL) repair ${ }^{12}$. REV7-deficient DT40 cells are hypersensitive to ICL-inducing reagents, such as cisplatin ${ }^{20}$. However, FAM $35 A^{-1-}$ and C20ORF196 ${ }^{-/-}$cells were not sensitive to cisplatin (Fig. 3a, b), suggesting that FAM35A and C20ORF196 are not required for the function of REV7 in ICL repair. These results are consistent with the biochemical data that REV7 forms a distinct complex with FAM35A and C20ORF196 from the $\mathrm{Pol} \zeta$ complex.

FAM35A and C20ORF196 block resection in the $B R C A 1^{-/-}$ cells. The 53BP1-RIF1-REV7 axis antagonizes BRCA1 to block DSB end resection and $\mathrm{HR}^{10}$. The absence of 53BP1, RIF1 or REV7 suppressed the PARP inhibitor (PARPi) sensitivity of the
BRCA1-deficient cells ${ }^{3-8,10}$. PARPi disrupts the repair of ssDNA breaks, which are converted to one-end DSBs during replication and are toxic to the cells deficient of HR proteins, such as BRCA1. We examined whether the REV7-FAM35A-C20ORF196 complex has similar function and generated $F A M 35 A^{-/} B R C A 1^{-/-}$ and C20ORF196 ${ }^{-/-}$BRCA1 ${ }^{-1-}$ double knockout DT40 cells. $B R C A 1^{-/-}$DT40 cells are hypersensitive to PARPi as reported ${ }^{4}$. Interestingly, the disruption of FAM35A or C20ORF196 gene suppressed the PARPi sensitivity of the $B R C A 1^{-/-}$cells (Fig. 5a, b). Moreover, analysis of chromosome aberration was consistent with these results (Fig. 5c), suggesting that FAM35A and C20ORF196 have BRCA1 antagonistic function in DSB repair similar to that of 53BP1, RIF1 and REV7. These results were further confirmed in human HCT116 cells (Supplementary Fig. 7a).

We further tested whether DNA end resection is altered in the absence of FAM35A or C20ORF196 in BRCA1-deficient cells. RPA binds to resected ssDNA and subsequently is phosphorylated by $\mathrm{ATM}^{21}$. Thus, RPA and phosphorylated RPA are widely used as a proxy for measuring resection. The signals of RPA and phosphorylated RPA were strongly decreased in the BRCA1deficient HCT116 cells treated with IR, but recovered when FAM35A or C20ORF196 was depleted (Supplementary Fig. 7b \pm e), suggesting that FAM35A and C20ORF196 suppresses end resection in the BRCA1 $1^{-/-}$cells. RAD51 will accumulate on $3^{\prime}$ ssDNA overhang after end resection during $\mathrm{HR}^{22}$. After X-ray treatment, BRCA1 $1^{-/}$DT40 cells showed significant decrease of RAD51 foci compared with wild-type cells (Fig. 5d, e). The disruption of FAM35A or C20ORF196 gene largely suppressed this defect in the $B R C A 1^{-/-}$DT40 cells (Fig. $5 \mathrm{~d}$, e), suggesting that FAM35A and C20ORF196 suppress end resection and HR in the BRCA1-deficient cells. Thus, FAM35A and C20ORF196 resemble 53BP1, RIF1 and REV7 to antagonize BRCA1 in DSB repair, indicating that REV7 performs its resection inhibition function through the REV7-FAM35A-C20ORF196 complex.

FAM35A has non-redundant function with $\mathrm{KU}$ to block resection. KU70/80 complex has strong binding affinity to blunt DSB ends and its binding protects ends from resection ${ }^{23-25}$. However, because the low binding affinity of KU70/80 to ssDNA,
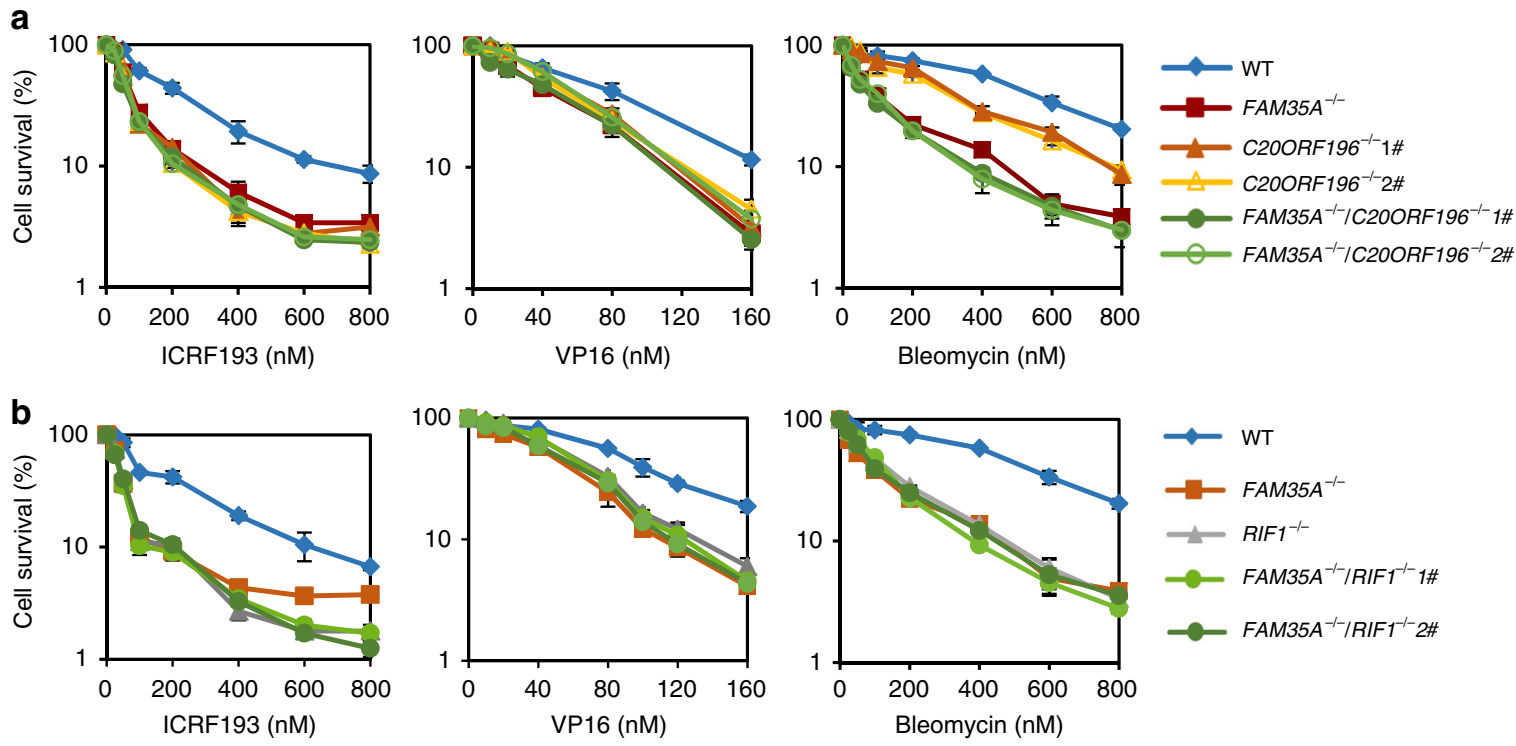

Fig. 4 FAM35A and C20ORF196 are in the same pathway with RIF1 to repair DSB. a, b Genetic interaction analysis of FAM35A with C20ORF196 (a) and RIF1 (b) in DT40 cells. The mean and s.d. from three independent experiments are shown 

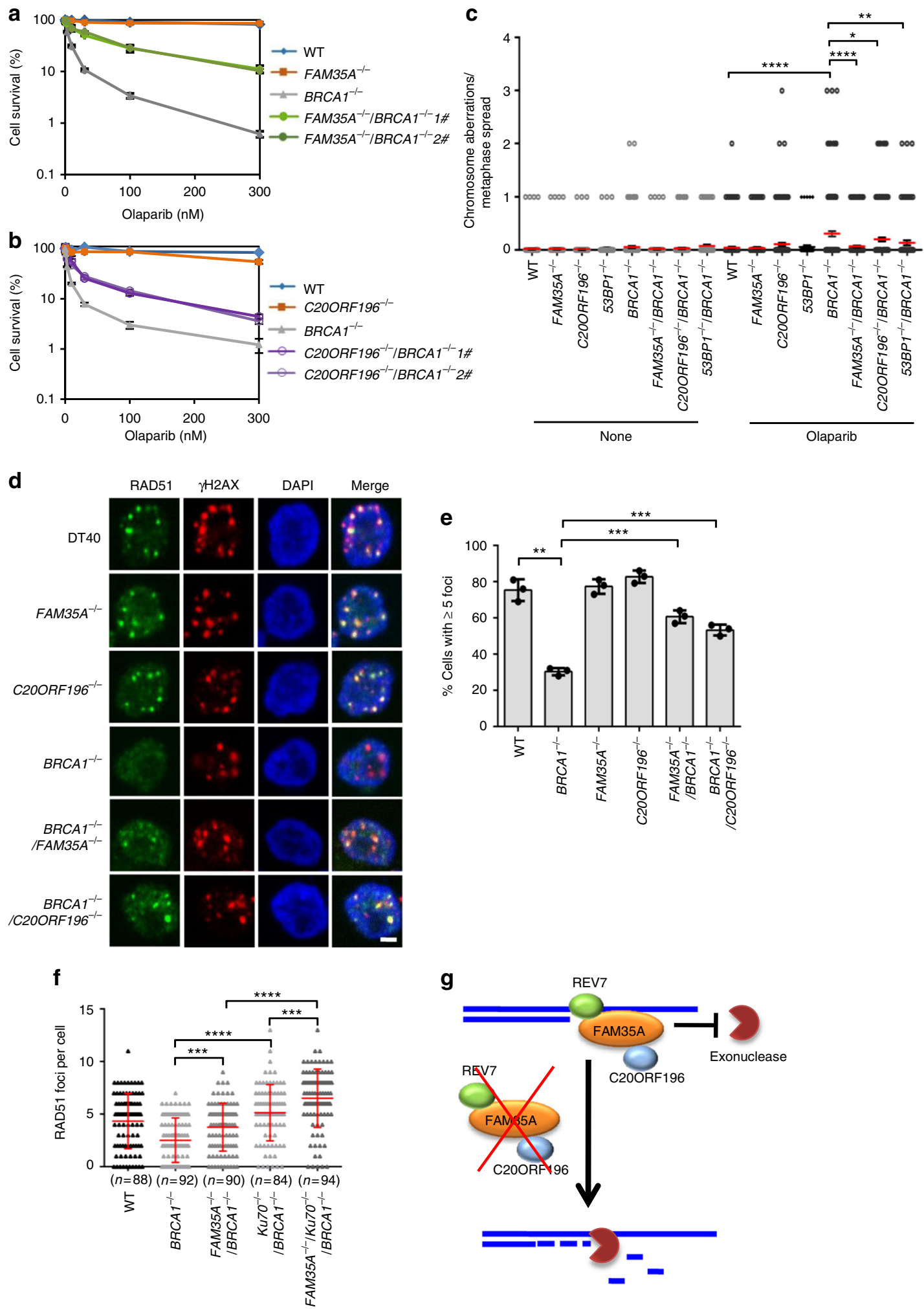

Fig. 5 FAM35A and C20ORF196 block resection in the BRCA1-deficient cells. a, b The absence of FAM35A (a) or C20ORF196 (b) suppressed the olaparib sensitivity of the BRCA1-deficient cells. The mean and s.d. from three independent experiments are shown. c Chromosome aberration of various DT40 cells. Cells were treated with or without $1 \mu \mathrm{M}$ olaparib for $8 \mathrm{~h}$ and then incubated with $100 \mathrm{ng} \mathrm{ml}^{-1}$ colcemid for $4 \mathrm{~h}$ before fixing. The mean and s.e.m. are shown. d, e Immunofluorescence (d) and its quantification (e) showing that FAM35A and C200RF196 block the end resection in the BRCA1-deficient cells. The DT40 cells were treated with $4 \mathrm{~Gy}$ X-ray and released $4 \mathrm{~h}$ before fixing. The mean and s.d. from three independent experiments are shown. Scale, $2 \mu \mathrm{m}$. f A scatter diagram shows RAD51 foci numbers in DT40 cells after X-ray treatment. The mean and s.d. are shown. $\mathbf{g}$ A model shows that REV7-FAM35A-C20ORF196 complex blocks resection through binding the 5 -recessed DSB ends. ${ }^{\star} p<0.05 ;{ }^{\star \star} p<0.01 ;{ }^{* \star *} p<0.001 ;{ }^{\star \star \star \star} p<0.0001$. Statistics was performed by two-tailed t-test 
it could not protect the $5^{\prime}$-recessed ends efficiently after partial resection ${ }^{1}$. We speculated that FAM35A may provide an opportunity to protect partially resected DSB ends when KU70/ 80 sequesters. Disruption of KU70 gene strongly rescues RAD51 foci in the BRCA1-deficient cells after X-ray treatment, suggesting that KU70/80 protects the DSB ends from resection (Fig. 5f). Interestingly, disrupting both KU70 and FAM35A genes led to a higher level of RAD51 foci than single gene knockout in the BRCA1-deficient cells (Fig. 5f). These results suggest that KU70/ 80 and FAM35A have non-redundant functions to prevent DNA end resection, consistent with our speculation.

\section{Discussion}

Altogether, our data identify a complex constituted of REV7-FAM35A-C20ORF196 as an important regulator of DSB repair pathway choice, which is downstream of 53BP1-RIF1 to antagonize BRCA1-dependent HR and to promote NHEJ. The complex is recruited to DSB sites mainly by RIF1 and binds preferably to ssDNA through the OB-folds of FAM35A (Fig. 2). FAM35A is structurally similar to POT1, which binds telomeric $3^{\prime}$ overhangs and blocks hyper-resection by Apollo/SNM1B exonuclease $^{15}$. We speculate that FAM35A works at DSB ends in a manner to POT1 at telomeres (Fig. 5g). FAM35A is able to inhibit unscheduled resection initiated by CtIP-MRN, limit overresection by EXO1/DNA2, or protect the recessed DSB ends, which might be a native product when DNA is damaged and is unable to be efficiently protected by KU70/80. A resectiondependent canonical NHEJ pathway was reported recently ${ }^{26}$. More recently, when our article was being prepared, several groups reported that FAM35A complex recruits CST(CTC1STN1-TEN1)-Pola complex to DSB sites to protect the broken ends ${ }^{27-33}$ through mimicking the action of POT1 at telomeres, consistent with our hypotheses.

\section{Methods}

Cell culture and transfection. U2OS and HEK293 cells were cultured in Dulbecco's modified Eagle's medium (DMEM) medium containing 10\% fetal bovine serum (FBS; Invitrogen). HEK293 suspension cells were cultured in SMM 293-TI medium (Sino Biological Inc.) supplemented with $1 \%$ Gibco FBS and $1 \%$ glutamine in an incubator with shaking at 140 r.p.m. The cell lines studied were obtained from the ATCC and are not among those listed as commonly misidentified by the International Cell Line Authentication Committee. All cell lines were subjected to mycoplasma testing twice per month and found to be negative. The identity of the cell lines was validated by Short Tandem Repeat profiling (ATCC) and by analysis of chromosome number in metaphase spreads.

HEK293 suspension cells were transfected with PEI. U2OS, HEK293T and HCT116 cells were transfected with FugeneHD (Promega). The small interfering RNAs (siRNAs) targeting Rif1 (RIF1siRNA in Supplementary Table 1), FAM35A (FAM35AsiRNA in Supplementary Table 1) and C20orf196 (C20ORF196siRNA in Supplementary Table 1), were transfected using RNAi MAX (Invitrogen). To produce the REV7 short hairpin RNA (Supplementary Table 1) virus, lentiviral plasmids were co-transfected into HEK293T cells using polyethylenimine (PEI). After 4 days, the supernatants containing the packaged lentivirus were harvested and stored at $-80^{\circ} \mathrm{C}$ until further use.

DT40 cells were gift from Dr. Shunichi Takeda and were cultured in RPMI1640 medium supplemented with $10 \%$ fetal calf serum, $1 \%$ chicken serum, $10 \mathrm{mM}$ HEPES and $1 \%$ penicillin-streptomycin mixture at $39.5^{\circ} \mathrm{C}, 5 \% \mathrm{CO}_{2}$. Transfection was performed by electroporation using the LonzaNucleofector $4 \mathrm{D}$. For selection, growth medium containing G418 $\left(2 \mathrm{mg} \mathrm{ml}^{-1}\right)$, puromycin $\left(0.5 \mu \mathrm{g} \mathrm{ml}^{-1}\right)$, Blasticidine $\left(25 \mu \mathrm{g} \mathrm{ml}^{-1}\right)$ or histidinol $\left(1 \mathrm{mg} \mathrm{ml}^{-1}\right)$ was used.

\footnotetext{
Antibodies. Anti-FAM35A rabbit polyclonal antibodies (1:500 for Westernblotting [WB]) are purchased from Abcam (ab136079), anti-C20ORF196 rabbit polyclonal antibodies (1:500 for WB) from Sigma-Aldrich (hpa040749), anti-REV7 rabbit polyclonal antibodies (1:2000 for WB) from BD Biosciences (612266), antiRAD51 rabbit polyclonal antibodies (1:200 for immunofluorescence [IF]) from Abcam (ab133534), anti-POLD3 rabbit polyclonal antibodies (1:2000 for WB) from Abnova (H00010714-M01), anti-GFP rabbit polyclonal antibodies (1:1000 for WB) from Proteintech Group (66002-1-Ig), anti- $\beta$-actin mouse monoclonal antibody (1:5000 for WB) from MBL (M177-3), anti-FLAG M2 monoclonal antibody (1:5000 for WB) from Sigma-Aldrich (F3165), anti-RPA32 rabbit monoclonal
}

antibody (1:500 for IF) from Abcam (ab76420) and anti-pRPA32 (S4/S8) rabbit polyclonal antibodies (1:500 for IF) from Bethyl (A300-245A).

Immunoprecipitation and MBP-pulldown. The complementary DNAs of REV7, FAM35A and C20ORF196 genes from hORFemone (V8.1) were transferred to mammalian expression destination plasmid pDEST26-FLAG with LR reaction (Invitrogen).

For immunoprecipitation (IP), expression plasmids were transiently transfected to HEK293 suspension cells with polyethyleneimine. Cells were harvested $64 \mathrm{~h}$ after transfection and pellets were directly lysed with NTEN buffer (20 mM Tris- $\mathrm{HCl}$ [pH 7.5], $150 \mathrm{mM} \mathrm{NaCl}, 10 \%$ glycerol, 0.5\% NP40, $10 \mathrm{mM} \mathrm{NaF}$ $1 \mathrm{mM}$ phenylmethylsulfonyl fluoride (PMSF), $1 \mu \mathrm{g} \mathrm{ml}^{-1}$ leupeptin, $1 \mu \mathrm{g} \mathrm{ml}^{-1}$ aprotinin). The lysates were ultra-centrifuged at $440,000 \times g$ for $15 \mathrm{~min}$ and then the supernatant was incubated with anti-Flag M2-conjugated agarose beads for $3-4 \mathrm{~h}$ at $4{ }^{\circ} \mathrm{C}$. The beads were washed four times with IP buffer $(20 \mathrm{mM}$ Tris- $\mathrm{HCl}$ [pH 7.5], $150 \mathrm{mM} \mathrm{NaCl}, \mathrm{MgCl}_{2} 5 \mathrm{mM}, 10 \%$ glycerol, $0.1 \% \mathrm{NP} 40,1 \mathrm{mM}$ DTT and $1 \mathrm{mM}$ PMSF) and then incubated with IP buffer containing $400 \mu \mathrm{g} \mathrm{ml}^{-1} 3 \mathrm{XFlag}$ peptide for $1-2 \mathrm{~h}$. Subsequently, the eluted complexes were analyzed by sodium dodecyl sulfate-polyacrylamide gel electrophoresis (SDS-PAGE) and mass spectrometry.

For MBP-pulldown, $30 \mu \mathrm{g}$ of pDEST26-MBP-FAM35A_RBD or pDEST26-MBP-MRE11 was transfected into $30 \mathrm{ml}$ of HEK293 suspension cells using polyethyleneimine. The cells were harvested after 3 days and lysed in $3 \mathrm{ml}$ of NTEN buffer. After ultra-centrifugation at $440,000 \times g$ for 15 min at $4{ }^{\circ} \mathrm{C}$, the supernatant was incubated with amylose resins at $4^{\circ} \mathrm{C}$ for $4 \mathrm{~h}$. The beads were washed four times with NTEN buffer, and eluted with $50 \mu \mathrm{l}$ of phosphate-buffered saline (PBS) containing $20 \mathrm{mg} \mathrm{ml}^{-1}$ Maltose.

Immunostaining and immunoblotting. DT40 cells were cultured to $0.8 \times 10^{6}$ cells $\mathrm{ml}^{-1}$ before the experiments. After washing with PBS, the cells were re-suspended with PBS containing $1 \%$ bovine serum albumin (BSA) and mixed with ninefold volume of PBS containing 3\% para-formaldehyde, $0.3 \%$ Triton, $0.5 \%$ BSA and $2 \%$ sucrose. The cells were immediately spun to slides with Cyto-spinner at $272 \times g$ for $8 \mathrm{~min}$. After washing three times with PBS with $0.05 \%$ Tween-20, the cells were blocked with 5\% BSA (Sigma) in PBS for 15 min. The primary antibodies were diluted in PBS containing 1\% BSA and incubated with the cells for $90 \mathrm{~min}$. After washing, secondary antibodies diluted in PBS containing 1\% BSA were added to the cells for $30 \mathrm{~min}$. The cells were washed three times and mounted with ProLong Gold antifade reagent with DAPI (Invitrogen). Images were acquired with an LSM710 confocal (Zeiss) using a $100 \times / 1.4$ NA objector.

For immunoblotting, primary antibodies were incubated for $1.5 \mathrm{~h}$ at room temperature in PBS with $0.05 \%$ Tween-20 and 5\% powder milk. Secondary peroxidase-coupled antibodies (1:5000; Jackson ImmunoResearch) were incubated at room temperature for $45 \mathrm{~min}$. Enhanced chemiluminescence was detected by using film. Uncropped immunoblots are available in Supplementary Fig. 8.

Laser microirradiation. U2OS or HEK293 cells expressing GFP-FAM35A or GFP-C20ORF196 were cultured at $37^{\circ} \mathrm{C}$ in $\mathrm{CO}_{2}$-independent medium (Invitrogen) containing $10 \%$ FBS in a temperature-controlled container in glass-bottom dishes (MatTek). Laser microirradiation was carried out with the Micro-Point Laser Illumination and Ablation System (ANDOR) coupled to a Leica DMI8 microscope with a $63 \times$ CS2 oil immersion objective. Images were acquired with ANDOR IQ3 software through an ANDOR IXON camera with ANDOR Dragonfly system.

Generation of RIF1 knockout HEK293 cells. RIF1-deficient HEK293 cells were generated using CRISPR. Briefly, guide sequences (RIF1sgRNA in Supplementary Table 1) were inserted into the $\mathrm{pX} 330$ vector $^{34}$. The guide sequence-containing pX330 plasmids were transfected into HEK293 cells and single colonies were picked after 8-10 days of incubation. The genomic fragments of the RIF1 gene were amplified by PCR using primers, RIF1g1 and RIF1g2 (Supplementary Table 1). The products were digested with T7 endonuclease. Colonies containing the expected PCR fragments were then sequenced and examined by western blotting.

Protein purification. FAM35A_C (381-904 aa) was fused with MBP-tag and $\mathrm{His}_{6}$-tag at $\mathrm{C}$-terminus and $\mathrm{N}$-terminus, respectively. Protein was expressed in E. coli (Transetta cells, TransGen) using pCPD9 vector. Cells were grown at $37^{\circ} \mathrm{C}$ until $\mathrm{OD} 600=0.8$, and were induced with $0.05 \%$ Isopropyl $\beta$-D-1thiogalactopyranoside at $16^{\circ} \mathrm{C}$ for $12 \mathrm{~h}$. The cell pellet from 12-liter culture was lysed by French Press in $200 \mathrm{ml}$ lysis buffer $(40 \mathrm{mM}$ Tris Cl, pH 8.0, $500 \mathrm{mM} \mathrm{NaCl}$ $25 \mathrm{mM}$ imidazole, $7 \%$ glycerol, $0.2 \mathrm{mM}$ DTT and $1 \mathrm{mM}$ PMSF, $1 \mu \mathrm{g} \mathrm{ml}^{-1}$ leupeptin and $1 \mu \mathrm{g} \mathrm{ml}^{-1}$ aprotinin). The lysate was centrifuged at 35,000 $\times \mathrm{g}$ for $30 \mathrm{~min}$ and the supernatant was incubated with $3 \mathrm{ml} \mathrm{Ni}$-beads (GE Healthcare) at $4{ }^{\circ} \mathrm{C}$ for $1 \mathrm{~h}$ The beads were washed four times with lysis buffer. The proteins were eluted with $15 \mathrm{ml}$ His-Elution Buffer $(20 \mathrm{mM}$ Tris Cl, pH 8.0, $160 \mathrm{mM} \mathrm{NaCl}, 400 \mathrm{mM}$ imidazole, $7 \%$ glycerol, $1 \mathrm{mM}$ DTT, $1 \mathrm{mM}$ PMSF, $1 \mu \mathrm{g} \mathrm{ml} l^{-1}$ leupeptin and $1 \mu \mathrm{g} \mathrm{ml}^{-1}$ aprotinin). The eluted proteins were incubated with $0.5 \mathrm{ml}$ Amylose resins (NEB) at $4{ }^{\circ} \mathrm{C}$ for $1 \mathrm{~h}$. After washing four times with washing buffer $(20 \mathrm{mM}$ Tris-HCl, 
$500 \mathrm{mM} \mathrm{NaCl}, 0.1 \%$ Triton X-100, $1 \mathrm{mM}$ DTT), protein was eluted with MBPElution Buffer (20 mM Tris Cl, pH 8.0, $160 \mathrm{mM} \mathrm{NaCl}, 7 \%$ glycerol, $1 \mathrm{mM}$ DTT and $30 \mathrm{mM}$ maltose). The protein was then concentrated, flash-frozen and stored at $-80^{\circ} \mathrm{C}$.

Gel-shift assay. The DNA substrates were made by annealing oligos H60f and H60r, H60f1/2 and H60r (Supplementary Table 1), respectively. The 5' ends of the oligo $\mathrm{H} 60 \mathrm{f}$ and $\mathrm{H} 60 \mathrm{f} 1 / 2$ were labeled with ${ }^{32} \mathrm{P}$ using T4 polynucleotide kinase before annealing. In all, $5 \mathrm{nM}^{32} \mathrm{P}$-labeled DNA subtracts and the indicated amount of proteins were incubated at $25^{\circ} \mathrm{C}$ in $10 \mu \mathrm{l}$ reaction buffer $(20 \mathrm{mM}$ HEPES at pH 7.5, $5 \mathrm{mM} \mathrm{MgCl}_{2}, 100 \mathrm{mM} \mathrm{KCl,} 1 \mathrm{mM} \mathrm{DTT}, 0.05 \%$ Triton X-100, $100 \mu \mathrm{g} \mathrm{ml}^{-1} \mathrm{BSA}$ and $5 \%$ glycerol) for $15 \mathrm{~min}$. Reaction mixture was loaded and resolved on a $5 \%$ Tris-borate-EDTA (TBE) gel.

Generation of the DT40 knockout strains. DT40 knockout constructs for FAM35A and C20ORF196 were generated as previously 35 using MultiSite Gateway Three-Flagment Vector Construction Kit. The $5^{\prime}$ and $3^{\prime}$ arms were amplified from genomic DNA using the primers FAM35A_5ARM1/FAM35A_5ARM2 and FAM35A3_ARM1/FAM35A3_ARM2 (for FAM35A; Supplementary Table 1), and C20ORF196_5ARM1/C20ORF196_5ARM2 and C20ORF196_3ARM1/ C20ORF196_3ARM2 (for C20ORF196; Supplementary Table 1). The knockout constructs were linearized before transfection. The primers FAM35A_g1/ FAM35A_g2 (for FAM35A; Supplementary Table 1) and C20ORF196_g1/ C20ORF196_g2 (for C20ORF196; Supplementary Table 1) were used for genomic DNA PCR. The generation of $R I F 1^{-/-}$and $B R C A 1^{-/-}$DT40 cells were described as previously $y^{5,36}$. FAM $35 A^{-1-} B R C A 1^{-1-}$ DT40 cells were generated by knockouting BRCA1 gene in FAM $35 A^{-/-}$cells. FAM $35 A^{-/-}$RIF1 $1^{-/-}$DT40 cells were generated by knockouting FAM $35 \mathrm{~A}$ gene in $\mathrm{RIF1}^{-/-}$cells. FAM $35 \mathrm{~A}^{-/-} \mathrm{Ku} 70^{-/-}$ $B R C A 1^{-1-}$ DT40 cells were generated by knockouting KU70 and BRCA1 genes successively.

Cell survival assay. In total, $1500-3000$ cells were plated into each well of 96-well plates with a range of doses of ICRF193, cisplatin or camptothecin (CPT). After 48 $\mathrm{h}$ of incubation, cells were pulsed with CellTiter 96 Aqueous One Solution Reagent (Promega) for $4 \mathrm{~h}$. Cell viability was measured by luminometer, and each dose point was measured in triplicate. For Bleomycin and VP16, a density of 300-1000 cells per well and a 72-h incubation were used.

Statistics. Statistics was performed by two-tailed $t$-test. The data were normally distributed and the variance between groups being statistically compared was similar. No statistical methods or criteria were used to estimate sample size or to include/exclude samples. The investigators were not blinded to the group allocation during the experiments.

\section{Data availability}

All relevant data are available from the authors.

Received: 12 April 2018 Accepted: 3 September 2018

Published online: 25 September 2018

\section{References}

1. Panier, S. \& Durocher, D. Push back to respond better: regulatory inhibition of the DNA double-strand break response. Nat. Rev. Mol. Cell Biol. 14, 661-672 (2013).

2. Panier, S. \& Boulton, S. J. Double-strand break repair: 53BP1 comes into focus. Nat. Rev. Mol. Cell Biol. 15, 7-18 (2014).

3. Bunting, S. F. et al. 53BP1 inhibits homologous recombination in Brca1deficient cells by blocking resection of DNA breaks. Cell 141, 243-254 (2010).

4. Escribano-Diaz, C. et al. A cell cycle-dependent regulatory circuit composed of 53BP1-RIF1 and BRCA1-CtIP controls DNA repair pathway choice. Mol. Cell 49, 872-883 (2013)

5. Feng, L., Fong, K. W., Wang, J., Wang, W. \& Chen, J. RIF1 counteracts BRCA1-mediated end resection during DNA repair. J. Biol. Chem. 288, 11135-11143 (2013).

6. Chapman, J. R. et al. RIF1 is essential for 53BP1-dependent nonhomologous end joining and suppression of DNA double-strand break resection. Mol. Cell 49, 858-871 (2013).

7. Zimmermann, M., Lottersberger, F., Buonomo, S. B., Sfeir, A. \& de Lange, T. 53BP1 regulates DSB repair using Rif1 to control 5' end resection. Science 339, 700-704 (2013).

8. Di Virgilio, M. et al. Rif1 prevents resection of DNA breaks and promotes immunoglobulin class switching. Science 339, 711-715 (2013).

9. Callen, E. et al. 53BP1 mediates productive and mutagenic DNA repair through distinct phosphoprotein interactions. Cell 153, 1266-1280 (2013).
10. Xu, G. et al. REV7 counteracts DNA double-strand break resection and affects PARP inhibition. Nature 521, 541-544 (2015).

11. Boersma, V. et al. MAD2L2 controls DNA repair at telomeres and DNA breaks by inhibiting 5' end resection. Nature 521, 537-540 (2015).

12. Lee, Y. S., Gregory, M. T. \& Yang, W. Human Pol zeta purified with accessory subunits is active in translesion DNA synthesis and complements Pol eta in cisplatin bypass. Proc. Natl. Acad. Sci. USA 111, 2954-2959 (2014).

13. Guo, R., Xu, D. \& Wang, W. Identification and analysis of new proteins involved in the DNA damage response network of Fanconi anemia and Bloom syndrome. Methods 48, 72-79 (2009).

14. Horvath, M. P. Structural anatomy of telomere OB proteins. Crit. Rev. Biochem. Mol. Biol. 46, 409-435 (2011).

15. Wu, P., Takai, H. \& de Lange, T. Telomeric $3^{\prime}$ overhangs derive from resection by Exol and Apollo and fill-in by POT1b-associated CST. Cell 150, 39-52 (2012).

16. Adachi, N., Suzuki, H., Iiizumi, S. \& Koyama, H. Hypersensitivity of nonhomologous DNA end-joining mutants to VP-16 and ICRF-193: implications for the repair of topoisomerase II-mediated DNA damage. J. Biol. Chem. 278, 35897-35902 (2003).

17. Xing, M. et al. Interactome analysis identifies a new paralogue of XRCC4 in non-homologous end joining DNA repair pathway. Nat. Commun. 6, 6233 (2015).

18. Wang, X., Takenaka, K. \& Takeda, S. PTIP promotes DNA double-strand break repair through homologous recombination. Genes Cells 15, 243-254 (2010).

19. $\mathrm{Xu}, \mathrm{Y}$. et al. $53 \mathrm{BP} 1$ and $\mathrm{BRCA} 1$ control pathway choice for stalled replication restart.eLife 6, e30523 (2017).

20. Okada, T. et al. Multiple roles of vertebrate REV genes in DNA repair and recombination. Mol. Cell. Biol. 25, 6103-6111 (2005).

21. Marechal, A. \& Zou, L. RPA-coated single-stranded DNA as a platform for post-translational modifications in the DNA damage response. Cell Res. 25, 9-23 (2015).

22. San Filippo, J., Sung, P. \& Klein, H. Mechanism of eukaryotic homologous recombination. Annu. Rev. Biochem. 77, 229-257 (2008).

23. Sun, J., Lee, K. J., Davis, A. J. \& Chen, D. J. Human Ku70/80 protein blocks exonuclease 1-mediated DNA resection in the presence of human Mre11 or Mre11/Rad50 protein complex. J. Biol. Chem. 287, 4936-4945 (2012).

24. Mimitou, E. P. \& Symington, L. S. Ku prevents Exo1 and Sgs1-dependent resection of DNA ends in the absence of a functional MRX complex or Sae2. EMBO J. 29, 3358-3369 (2010).

25. Shim, E. Y. et al. Saccharomyces cerevisiae Mre11/Rad50/Xrs2 and Ku proteins regulate association of Exo1 and Dna2 with DNA breaks. EMBO J. 29, 3370-3380 (2010).

26. Biehs, R. et al. DNA double-strand break resection occurs during nonhomologous end joining in G1 but is distinct from resection during homologous recombination. Mol. Cell 65, 671-684 e675 (2017).

27. Ghezraoui, H. et al. 53BP1 cooperation with the REV7-shieldin complex underpins DNA structure-specific NHEJ. Nature 560, 122-127 (2018).

28. Noordermeer, S. M. et al. The shieldin complex mediates 53BP1-dependent DNA repair. Nature 560, 117-121 (2018).

29. Dev, H. et al. Shieldin complex promotes DNA end-joining and counters homologous recombination in BRCA1-null cells. Nat. Cell Biol. 20, 954-965 (2018).

30. Tomida, J. et al. FAM $35 \mathrm{~A}$ associates with REV7 and modulates DNA damage responses of normal and BRCA1-defective cells.EMBO J. 37, e99543 (2018).

31. Gupta, R. et al. DNA repair network analysis reveals Shieldin as a key regulator of NHEJ and PARP inhibitor sensitivity. Cell 173, 972-988 e923 (2018).

32. Mirman, Z. et al. 53BP1-RIF1-shieldin counteracts DSB resection through CST- and Polalpha-dependent fill-in. Nature 560, 112-116 (2018).

33. Barazas, M. et al. The CST complex mediates end protection at double-strand breaks and promotes PARP inhibitor sensitivity in BRCA1-deficient cells. Cell Rep. 23, 2107-2118 (2018).

34. Cong, L. et al. Multiplex genome engineering using CRISPR/Cas systems. Science 339, 819-823 (2013).

35. Iiizumi, S. et al. Simple one-week method to construct gene-targeting vectors: application to production of human knockout cell lines. Biotechniques 41, 311-316 (2006)

36. $\mathrm{Xu}, \mathrm{D}$. et al. Rif1 provides a new DNA-binding interface for the Bloom syndrome complex to maintain normal replication. EMBO J. 29, 3140-3155 (2010).

\section{Acknowledgements}

We thank Weidong Wang for his advice and revisions to the manuscript. We thank the Core Facility of Life Sciences, Peking University for assistance with the imaging. This work was supported in part by the National Natural Science Foundation of China (31870807, 81672773 and 31661143040). 


\section{Author contributions}

S.G., S.F., S.N, J.L., H.Z., Y.X., J.S. and K.L. carried out experiments. Q.L., R.G. and D.X. designed experiments and interpreted the results. S.G. and D.X. wrote the manuscript.

\section{Additional information}

Supplementary Information accompanies this paper at https://doi.org/10.1038/s41467018-06407-7.

Competing interests: The authors declare no competing interests.

Reprints and permission information is available online at http://npg.nature.com/ reprintsandpermissions/

Publisher's note: Springer Nature remains neutral with regard to jurisdictional claims in published maps and institutional affiliations. (c) (i) Open Access This article is licensed under a Creative Commons Attribution 4.0 International License, which permits use, sharing, adaptation, distribution and reproduction in any medium or format, as long as you give appropriate credit to the original author(s) and the source, provide a link to the Creative Commons license, and indicate if changes were made. The images or other third party material in this article are included in the article's Creative Commons license, unless indicated otherwise in a credit line to the material. If material is not included in the article's Creative Commons license and your intended use is not permitted by statutory regulation or exceeds the permitted use, you will need to obtain permission directly from the copyright holder. To view a copy of this license, visit http://creativecommons.org/ licenses/by/4.0/.

(c) The Author(s) 2018 